\title{
Involved radial margins: Mountain or molehill?
}

\author{
Wayne L. Hofstetter, MD
}

See related article on pages 548-55.

The significance of an isolated positive radial margin $(+\mathrm{RM})$ after esophagectomy has received quite a bit of attention in the literature during the past decade, with at least 8 articles published since the changes in 2010 the seventh edition of the American Joint Committee on Cancer's staging manual. The importance of the time frame of publication relates to the consequence of an involved radial margin relative to nodal disease burden, a powerful indicator of prognosis and a frequent covariable. Before the seventh edition, the number of involved nodes was not accounted for in the esophageal staging system, and studies that used disease stage alone were therefore not adequately granular to compare outcomes. Recent changes in practice patterns favoring preoperative therapy and the adjustments in the staging system have prompted several groups to reevaluate the implications of a resection resulting in an isolated positive radial margin.

In their article in this issue of the Journal, Gilbert and colleagues $^{1}$ describe their review of 154 patients, some treated with preoperative therapy $(57 / 154 ; 37 \%)$ and others with surgery alone. Patients with tumor at the radial margin, defined as a positive margin according to the College of American Pathologists criteria, were compared with those who had an $\mathrm{R} 0$ resection. The patients were analyzed for long-term outcomes, with the hypothesis that patients with $+\mathrm{RM}$ would be at higher risk for locoregional failure and shorter overall survival. Gilbert and colleagues ${ }^{1}$ report that local recurrence was in fact not affected by $+\mathrm{RM}$; however, patients with $+\mathrm{RM}$ did have higher incidence of distant failure and worse overall survival. They conclude that $+\mathrm{RM}$ is a marker for poor prognosis and discuss the possibility that adjuvant chemotherapy would be useful in these circumstances. Unfortunately, despite the fact that a large percentage of the patients with +RM in the study received adjuvant therapy, no data were supplied to compare the outcomes of those with versus without adjuvant therapy.

From the Department of Thoracic Surgery, MD Anderson Cancer Center, Houston, Tex.

Disclosures: Author has nothing to disclose with regard to commercial support.

Received for publication Oct 14, 2014; accepted for publication Oct 14, 2014; available ahead of print Nov 6, 2014.

Address for reprints: Wayne L. Hofstetter, MD, Department of Thoracic Surgery, MD Anderson Cancer Center, 1515 Holcombe Blvd, Unit 445, Houston, TX 77030

(E-mail: whofstetter@mdanderson.org).

J Thorac Cardiovasc Surg 2015;149:556-7

0022-5223/\$36.00

Copyright (c) 2015 by The American Association for Thoracic Surgery

http://dx.doi.org/10.1016/j.jtcvs.2014.10.066
There is fairly strong corroborating evidence (retrospective case series and meta-analyses) to support the statement that a patient with $+\mathrm{RM}$ who has not received preoperative therapy is at higher risk for disease-related mortality. On the other hand, my own group ${ }^{2}$ published a similar article concluding that a $+\mathrm{RM}$ after preoperative therapy is more likely to represent an indicator of tumor biology than a specific impetus to further local therapies. Given that apparent discrepancy, a valid question is whether modalities that affect the incidence of an R0 resection have an effect on survival for an entire cohort or just an isolated group of responders. If either an en bloc resection, preoperative therapy, or both could result in a superior $\mathrm{R} 0$ resection rate relative to other therapies, would the outcomes improve or simply balance out given the additional nontherapeutic treatment risk? Whereas I fully agree with Gilbert and colleagues that comparison between studies can be difficult, there are some apparent distinctions that are worthy of discussion.

The +RM rate by College of American Pathologists criteria for this article is quoted at $19 \%$, almost exclusively occurring within patients with stage III disease. In contrast, we reported on 160 patients, all with adenocarcinoma, all treated with preoperative chemoradiation, and $86 \%$ undergoing resection by a transthoracic operation. In that cohort of consecutive patients, only $5 \%$ fulfilled the definition of the College of American Pathologists for $+\mathrm{RM}$. Another noted difference is that our entire series had ypT3 or higher disease, the group that is at highest risk for a + RM. We concluded that in an unmatched group of patients, + RM was an indicator of a biologically more aggressive cancer but was not an independent predictor of outcome.

Of some interest are the qualifying statements in Gilbert and colleagues' discussion ${ }^{1}$ indicating that bigger surgery (en bloc resection) and preoperative therapy would perhaps improve the R0 resection rate, although at the expense of patient risk. What seems to be most important in any debate on surgical therapy is that the role of resection is to facilitate locoregional disease control. When the disease presents in locally advanced stages, however, we can predict that attaining control and the potential for cure can be difficult. In Gilbert and colleagues' cohort $^{1}$ the highest risk group (stage III) had a $33 \% \mathrm{R} 1$ resection rate, and they conclude that the finding that $\mathrm{R} 1$ resection portends a negative prognosis indicates that perhaps one of our goals should be to maximize the chances of an R0 resection. 
Retrospective and randomized studies have shown that preoperative therapy, bigger surgery, or both achieve exactly that. Irrespective of local recurrence, an event that occurs too infrequently to derive any conclusions in these articles, incomplete resection stands out as failure of overall therapy. Further research that will define which individual patients actually derive survival benefit from therapy, including resection, will lead us to better therapy selections. Until then, I am sticking with preoperative therapy and bigger surgery for patients at the highest risk for incomplete resection.

\section{References}

1. Gilbert S, Martel AB, Seely AJ, Maziak DE, Shamji FM, Sundaresan SR, Villeneuve PJ. Prognostic significance of a positive radial margin after esophageal cancer resection. J Thorac Cardiovasc Surg. 2015;149:548-55.

2. Harvin JA, Lahat G, Correa AM, Lee J, Maru D, Ajani J, et al. Neoadjuvant chemoradiotherapy followed by surgery for esophageal adenocarcinoma: significance of microscopically positive circumferential radial margins. J Thorac Cardiovasc Surg. 2012;143:412-20. 